\title{
CHARACTERIZATIONS FOR VECTORIAL PREQUASI-INVEX TYPE FUNCTIONS VIA JENSEN TYPE INEQUALITIES
}

\begin{abstract}
RONG HU
Abstract. The purpose of this paper is to derive some criteria for vectorial prequasi-invex type functions via Jensen type inequalities. It is shown that a Jensen type inequality is sufficient and necessary for a vector function to be prequasi-invex under the condition of lower levelclosedness, cone lower semicontinuity, cone upper semicontinuity and semistrict prequasi-invexity, respectively. Analogous results are established for vectorial semistrictly prequasi-invex functions and vectorial strictly prequasi-invex functions.
\end{abstract}

Mathematics subject classification (2010): 26B25, 90C29.

Keywords and phrases: Jensen type inequalities, prequasi-invexity, semistrict prequasi-invexity, strict prequasi-invexity, lower level-closedness, cone lower semicontinuity, cone upper semicontinuity.

\section{REFERENCES}

[1] X. M. YANG, Convexity of semi-continuous functions, Opsearch 31 (4) (1994), 309-317.

[2] R. N. MukHeRJEe AND L. V. Reddy, Semicontinuity and quasiconvex functions, J. Optim. Theory Appl. 94 (3) (1997), 715-726.

[3] X. M. YAng, X. Q. YAng, And K. L. TeO, Characterizations and applications of prequasi-invex functions, J. Optim. Theory Appl. 110 (3) (2001), 645-668.

[4] H. Z. LuO AND Z. K. XU, On characterizations of prequasi-invex functions, J. Optim. Theory Appl. 120 (2) (2004), 429-439.

[5] M. A. Hanson, On sufficiency of the Kuhn-Tucker conditions, J. Math. Anal. Appl. 80 (2) (1981), $545-550$.

[6] T. WeIR And B. Mond, Pre-invex functions in multiple objective optimization, J. Math. Anal. Appl. 136 (1) (1988), 29-38.

[7] T. WEIR AND V. JEYAKUMAR, A class of nonconvex functions and mathematical programming, Bull. Austral. Math. Soc. 38 (2) (1988), 177-189.

[8] R. PINI, Invexity and generalized convexity, Optimization 22 (4) (1991), 513-525.

[9] D. T. LuC, Theory of Vector Optimization, Leture Notes in Economics and Mathematical Systems, No. 319, Springer-Verlag, Berlin, (1989).

[10] X. M. YANG, X. Q. YANG, AND K. L. TEO, Generalized invexity and generalized invariant monotonicity, J. Optim. Theory Appl. 117 (3) (2003), 607-625.

[11] X. M. Yang And D. Li, Semistrictly preinvex functions, J. Math. Anal. Appl. 258 (1) (2001), $287-$ 308.

[12] S. R. Mohan And S. K. NeOgy, On invex sets and preinvex functions, J. Math. Anal. Appl. 189 (3) (1995), 901-908.

[13] T. TANAKA, Generalized semicontinuity and existence theorems for cone saddle points, Appl. Math. Optim. 36 (3) (1997), 313-322.

[14] V. JeYAKUMAR AND H. WOLKOWICZ, Zero duality gaps in infinite-dimensional programming, J. Optim. Theory Appl. 67 (1) (1990), 87-108. 